\title{
THE DIAGNOSTIC VALUE OF ANTIBODIES TO NEUTROPHIL ELASTASE IN NAFLD AND COPD PATIENTS
}

\author{
Mariia Derbak \\ Doctor of Medical Sciences, Professor, Head of the Department of Faculty Therapy, \\ Uzhhorod National University, Ukraine \\ e-mail: morika1415@gmail.com,orcid.org/0000-0003-4791-4080
}

Iryna Khramtsova

Ph.D., Assistant Professor, Uzhhorod National University, Ukraine e-mail: bukovskaira@gmail.com,orcid.org/0000-0002-5603-5515

\begin{abstract}
Summary
This article presents data on the influence of enzymatic activity of serum on the processes of liver fibrosis in patients with non-alcoholic fatty liver disease (NAFLD) and chronic obstructive pulmonary disease (COPD). Clinical, biochemical, immunological research methods and enzyme-linked immunosorbent assay (ELISA) were used in the work. All patients were determined for the degree of liver fibrosis using a non-invasive method - Fibromax. It was found that in patients with NAFLD and COPD with frequent exacerbations, the levels of CRP, IL-6, TNF- $\alpha$ and neopterin are significantly higher than in patients who had one or no exacerbation of COPD. Stably high levels of systemic inflammation markers of TNF- $\alpha$, neopterin, CRP lead to the activation of TGF- $\beta$, which increases with the deepening stage of liver fibrosis. The average values of IgG antibodies to neutrophil elastase significantly increase depending on the stage of fibrotic changes in the liver and the activity of inflammatory changes in it, with the highest concentration in fibrosis F2. In F3-4 fibrosis, the levels of antibodies to elastase are reduced, although they remain higher than the control values. Significant increase IgG antibody levels to neutrophil elastase depending on the stage of fibrosis and the activity of inflammatory changes indicates the role of serum enzymatic activity in the mechanisms of formation of more severe stages of NAFLD and can be considered as additional diagnostic markers.
\end{abstract}

Keywords: liver fibrosis, hepatic steatosis, enzymatic activity of blood serum.

DOI: https://doi.org/10.23856/4819

\section{Introduction}

There is a strong justification for supporting the hypothesis of an increase in the prevalence of nonalcoholic fatty liver disease (NAFLD) in patients with chronic obstructive pulmonary disease (COPD) (Chan, Stanley et al., 2019). Frequent combination of COPD and NAFLD is due to the common links of pathogenesis, namely the presence of systemic inflammation. There is strong evidence that pneumonia during COPD leads to an increase the number of biomarkers associated with neutrophilic inflammation (MMP9, elastase, calprotectin) and proinflammatory cytokines (IL-6, IL- $1 \beta$, IFN- $\alpha$, CRP, TNF-a,) not only "in the lungs and bronchi", but also in the peripheral blood, which indicates the presence of a systemic inflammatory reaction or systemic inflammation and plays an important role in pathophysiology of COPD and its comorbidities such as metabolic syndrome (Yazici et al.,2020; Huang et al., 2019). Several studies have reported that frequent exacerbations of COPD potentiate the progression 
of NAFLD with high inflammatory activity and increased hepatic steatosis. Recently, there are conflicting data in the literature on the role of neutrophils in the human body. Traditionally, neutrophils are recognized as key components of the innate immune system, which exhibit antimicrobial functions and participate in the regulation of the adaptive immune response (Xu et al., 2020) and are the first-line protection in acute inflammation caused by infection (Amulic et al., 2020). However, other data show that neutrophils are heterogeneous and may be involved in tissue damage, as their activators may be not only infectious causes, but also other cell damage (Lehman et al., 2020). There have been isolated reports that infiltration of neutrophils into the liver with their autocrine, paracrine, and immune properties is a major pathological feature of various liver diseases, including nonalcoholic steatohepatitis (NASH), viral hepatitis, and alcoholic liver disease (Liu et al., 2021). That is, the function of neutrophils in various liver diseases can be not only protective but also damaging. Neutrophils release various serine proteases, including neutrophil elastase (NE), to activate hepatic macrophages and secrete chemokines and cytokines to induce peripheral blood macrophage aggregation and Kupffer cell proliferation (Wu et al., 2020). It has also been found that NE leads to activation of metalloproteinases (MMPs), connective tissue remodeling, activation of immune resistance mechanisms and is a regulator in maintaining the activity of pro- and anti-inflammatory cytokines. However, the exact mechanisms of the pathogenesis of the transition from steatosis to fibrosis in patients with NAFLD under the condition of comorbidity of COPD have not been fully established.

Despite the large number of studies to date, there is no effective treatment of NAFLD, which emphasizes the need for further study of the main pathogenetic mechanisms underlying the development and progression of hepatic steatosis in NASH in patients with COPD. The role of serum enzymatic activity, in particular neutrophil elastase, which may be important for the diagnosis and treatment of patients in this category, needs further study.

The aim of research was to establish the relationship between levels of antibodies to neutrophil elastase and markers of liver fibrosis in patients with NAFLD under the condition of comorbidity with COPD.

\section{Materials and methods of research}

The following parameters were studied - TNF-a, IL-6, neopterin, tissue growth factor (TGF) $-\beta$, IgG antibodies to neutrophil elastase and tissue inhibitor of metalloproteinases-1 (TIMP-1) in the serum of 82 patients diagnosed with NAFLD and COPD of groups B and C. Among the surveyed were $61.0 \%$ (50) men and $40.0 \%$ (32) women. The mean age of patients was $57.8 \pm 1.5$ years.

Depending on the nosology, patients were divided into two groups: group 1 (main, $\mathrm{n}=52$ ) - NAFLD + COPD and Group 2 (comparison, $\mathrm{n}=30$ ) - patients with NAFLD. Taking into account the frequency of exacerbations of COPD, group 1 was divided into: group 1a $(\mathrm{n}=22)-$ patients with NAFLD + COPD group II and group $1 \mathrm{~b}(\mathrm{n}=30)-$ patients with NAFLD + COPD group II C. The average exacerbation in group $1 \mathrm{a}-1.02 \pm 0.22$ and in group $1 b-2.52 \pm 0.25$. The groups were representative by age, sex and severity of the disease. The control group consisted of 30 healthy individuals (mean age $35.3 \pm 1.4$ years). Patients were prescribed basic therapy for COPD in accordance with the order of the Ministry of Health of Ukraine №555 from 27.06.13 (fenoterol / ipratropium bromide 50/20 in one dose after 8 hours).

The studies were performed in the exacerbation phase of COPD and the remission phase - after 3 months after discharge from the hospital. Studies were conducted with the informed consent of patients, and their methodology complies with the Helsinki Declaration 
of 1975 and its 1983 revision approved by the local Research Commission (Protocol №1 from 10.01.2020).

The inclusion criteria in the study confirmed the diagnosis of chronic obstructive pulmonary disease (GOLD II) and age over 40 and less than 70 years and / or NAFLD. Exclusion criteria were the presence of markers of viral hepatitis $\mathrm{B}$ and $\mathrm{C}$, markers of autoimmune hepatitis / cross syndrome (anti-LKM-1, anti-SLA and anti-LC-1) and HIV infection, alcohol consumption, toxic liver damage and patient rejection. from research.

The diagnosis of COPD was made according to the International Classification of Diseases-10 revision and confirmed by spirometry, which recorded a decrease in the ratio of FEV1/ FVC $<0.7(70 \%)$ after taking a bronchodilator. The duration of the disease, the frequency of exacerbations of COPD during the last year was determined by retrospective study of the anamnesis (order of the Ministry of Health of Ukraine №555 from 27.06.13).

The diagnosis of NAFLD was established according to the unified clinical protocol "Non-alcoholic steatohepatitis" (2014) and the adapted clinical guideline based on the evidence "Non-alcoholic fatty liver disease" (2012), according to the recommendations of the European Association for the Study of the Liver (EASL).

Enzyme-linked immunosorbent assay (ELISA) was used to determine levels of C-reactive protein (CPR), concentrations of TNF-a, IL-6, neopterin and IgG antibodies to neutrophil serum elastase. The results of the studies were taken into account on an automatic enzymelinked immunosorbent assay "STATFAX" according to the instructions included with the reagent kits Diagnostics Biochem Canada and DRG (USA). The functional state of the liver was assessed by biochemical analysis of blood - total bilirubin and its fractions, total protein and protein fractions, the activity of serum cytolytic enzymes alanine aminotransferase (ALT) and aspartate aminotransferase (AST) and activity of cholestatic enzymes (alkaline phosphatase and $\gamma$-glutamyltranspeptidase), the level of urea, creatinine, glucose, which was determined using an automatic biochemical analyzer and original reagents ChemWell, Awareness Technology INC (USA). The lipid composition of the blood was characterized by the concentration of total cholesterol, low-density lipoprotein cholesterol (LDL cholesterol), high-density lipoprotein cholesterol (HDL cholesterol) and triglycerides (TG), using kits "Bio-La-ChechA". Indicators of the general analysis of blood were defined according to the standard method using an automatic hematology analyzer BC-2800 (Mindray, China).

To determine the stage of steatosis and liver fibrosis used a non-invasive method of diagnosis - FibroMax, which includes: FibroTest, ActiTest, SteatoTest, AshTest, NashTest. The study is based on a comprehensive analysis of 10 biochemical parameters: alpha-2-Macroglobulin, haptoglobin, Apolipoprotein-AI, gamma-glutamyl transpeptidase, total bilirubin, ALT, AST, blood glucose, TG, cholesterol. All general clinical, biochemical and immunological tests were performed in certified laboratories, all medical equipment was under metrological control.

All patients underwent abdominal ultrasound examination according to the conventional method, which was performed on a Philips HDI-1500 with a scanning sensor with a frequency of 3.5 MHz. Depending on the ultrasound picture of fatty infiltration of the liver and the inflammatory process in all patients, steatosis was assessed as minimal, moderate and severe. To assess the trophological status of patients, height, weight, waist circumference, and body mass index (BMI) were determined, which was calculated according to the generally accepted formula. The BMI of $18.5-24.9 \mathrm{~kg} / \mathrm{m} 2$ was considered the norm. According to the criteria of the International Diabetes Federation (IDF) in 2005 to determine the criteria of abdominal or central obesity were assessed using waist circumference (WC), waist-to-hip ratio (WHR) and waist-to-height ratio (WHTR). The presence of abdominal obesity in patients was confirmed 
by anthropometric data, namely: an increase in waist circumference (WC) in men from 94 to $130 \mathrm{~cm}$, on average up to $(102.8 \pm 1.2) \mathrm{cm}$, in women from 82 to $142 \mathrm{~cm}$, on average up to $(93.1 \pm 2.0) \mathrm{cm}$; as well as measuring the waist-to-hip ratio (WHR) and body mass index (BMI). Patients were diagnosed with abdominal obesity in accordance with generally accepted criteria for certain of central obesity indexes.

For statistical processing of the obtained data, parametric and nonparametric methods (Kruskal-Wallis test, Mann-Whitney test; Pearson's linear correlation coefficient, Student's t-test) were used in the SPSS 11.5 package environment. The mean values, standard errors and significance of differences that were considered probable at $\mathrm{p}<0.05$ were estimated.

\section{Results}

Analyzing the data obtained, it was found that increased levels of markers of chronic inflammation were observed in all subjects. However, it is noteworthy that in patients with comorbid conditions, the increase in each indicator was significantly higher than in patients with one nosology. The levels of some markers depended on the frequency of exacerbations of COPD (Table 1).

Table 1

The average values of individual blood parameters in the examined patients

\begin{tabular}{|c|c|c|c|c|}
\hline \multirow{3}{*}{ Parameters } & \multicolumn{4}{|c|}{ Groups } \\
\hline & \multicolumn{2}{|c|}{$\begin{array}{l}\text { NAFLD + COPD } \\
(\text { main group, } n=52)\end{array}$} & \multirow{2}{*}{$\begin{array}{c}\text { NAFLD } \\
\text { comparison } \\
\text { group }(n=30)\end{array}$} & \multirow{2}{*}{$\begin{array}{l}\text { Control group } \\
\text { of healthy } \\
\text { individuals } \\
(\mathbf{n}=\mathbf{3 0})\end{array}$} \\
\hline & $\underset{n=22}{\operatorname{group} 1 a}$ & $\underset{n=30}{\operatorname{group} 1 b}$ & & \\
\hline CRP, mg / 1 & $16,4 \pm 1,7^{* \bullet}$ & $22,5 \pm 2,5_{* *}^{\bullet *}$ & $3,6 \pm 0,3$ & $2,7 \pm 0,9$ \\
\hline $\mathrm{ESR}, \mathrm{mm} / \mathrm{h}$ & $15,3 \pm 0,3^{* \bullet}$ & $18,0 \pm 0,3^{\bullet *}$ & $8,2 \pm 0,3$ & $8,5 \pm 2,8$ \\
\hline TNF-a, pg / ml & $207,5 \pm 10,3^{*}$ & $565,3 \pm 12,5_{* *}^{\bullet *}$ & $175,6 \pm 5,4^{*}$ & $9,5 \pm 0,7$ \\
\hline IL-6, pg / ml & $15,4 \pm 1,3^{* \bullet}$ & $19,5 \pm 0,8^{\bullet *}$ & $7,8 \pm 1,7^{*}$ & $3,9 \pm 1,2$ \\
\hline Neopterin, nmol/ 1 & $147,3 \pm 0,3^{*}$ & $428,6 \pm 1,5_{* *} \bullet$ & $109,5 \pm 1,8^{*}$ & $7,2 \pm 1,2$ \\
\hline TGF- $\beta, \mathrm{pg} / \mathrm{ml}$ & $357,3 \pm 58,9^{*}$ & $429,8 \pm 95,2_{* *}^{\bullet}$ & $245,4 \pm 15,7^{*}$ & $154,0 \pm 22,7$ \\
\hline TIMP-1, ng / ml & $624,0 \pm 127,3^{* \bullet}$ & $733,4 \pm 119,5_{* *}^{\bullet *}$ & $458,6 \pm 76,3^{*}$ & $122,0 \pm 17,3$ \\
\hline $\begin{array}{l}\text { Antibodies to IgG } \\
\text { Neutrophil elastase, } \\
\mathrm{U} / \mathrm{ml}\end{array}$ & $32,2 \pm 3,5^{*}$ & $41,2 \pm 5,8^{\bullet}$ & $6,2 \pm 1,8^{*}$ & $3,2 \pm 0,8$ \\
\hline $\begin{array}{l}\text { Total cholesterol } \\
\text { (TC), mmol / } 1\end{array}$ & $6,2 \pm 0,5^{*}$ & $5,9 \pm 0,7^{*}$ & $5,5 \pm 0,4^{*}$ & $4,2 \pm 0,7$ \\
\hline $\begin{array}{l}\text { Triglycerides (TG) } \\
\mathrm{mmol} / \mathrm{l}\end{array}$ & $3,2 \pm 0,4^{*} \bullet$ & $2,8 \pm 0,3^{*}$ & $2,6 \pm 0,2^{*}$ & $1,3 \pm 0,3$ \\
\hline ALAT, IU / 1 & $89,8 \pm 12,4^{* \bullet}$ & $75,5 \pm 18,2^{*}$ & $65,3 \pm 10,2^{* *}$ & $20,5 \pm 3,4$ \\
\hline BMI, kg / m2 & $28,5 \pm 0,7^{*}$ & $26,5 \pm 0,5$ & $27,5 \pm 0,3$ & $22,5 \pm 0,4$ \\
\hline $\begin{array}{l}\text { Waist-to-hip ratio } \\
\text { (WHR) }\end{array}$ & $1,35 \pm 0,2^{*}$ & $1,20 \pm 0,3^{*}$ & $1,15 \pm 0,1^{*}$ & $0,78 \pm 0,05$ \\
\hline
\end{tabular}

Notes: Significance of the difference: ${ }^{*}-$ with the control group; ${ }_{* *}-$ with group 1a;

- - with group 2 (the indicator is calculated by the Mann-Whitney test, $\mathrm{p}<0,05$ ) 
The highest values of CRP were in the group of patients with NAFLD + COPD with frequent exacerbations ( 2 or more exacerbations per year), which required hospitalization and exceeded the norm by 8.3 times. TNF-a and neopterin levels in patients with frequent exacerbations of COPD with hospitalization were 2.7 times and 1.9 times higher than in patients with a small number of exacerbations $(\mathrm{p}<0.05)$ and 3.2 and 3.9 times respectively, higher than in patients with NAFLD without COPD. Patients with NAFLD combined with COPD, proinflammatory cytokines TNF- $\alpha$, neopterin, and CRP levels are major in maintaining the inflammatory process during exacerbations. There was also a positive correlation between TNF- $\alpha$ and neopterin with the number of segmental neutrophils and levels of antibodies to neutrophil elastase $(\mathrm{r}=0.38 ; \mathrm{p}<0.05$ and $\mathrm{r}=0.42 ; \mathrm{p}<0.05)$.

It should be noted that the concentration of $\operatorname{IgG}$ antibodies to neutrophil elastase is significantly higher in all patients with NAFLD, regardless of the frequency of exacerbations of COPD. However, it was found that the average values of IgG antibodies to neutrophil elastase significantly increase depending on the stage of fibrotic changes in the liver and the activity of inflammation $(p<0.05)$, with its highest concentration in fibrosis F2 and directly correlate with BMI, cholesterol concentration, triglycerides and alanine aminotransferase $(r=0.43 ; p<0.05$ and $\mathrm{r}=0.45 ; \mathrm{p}<0.05)$. In fibrosis F3-4, the levels of antibodies to elastase are reduced, although they remain higher than the control values (Fig. 1)

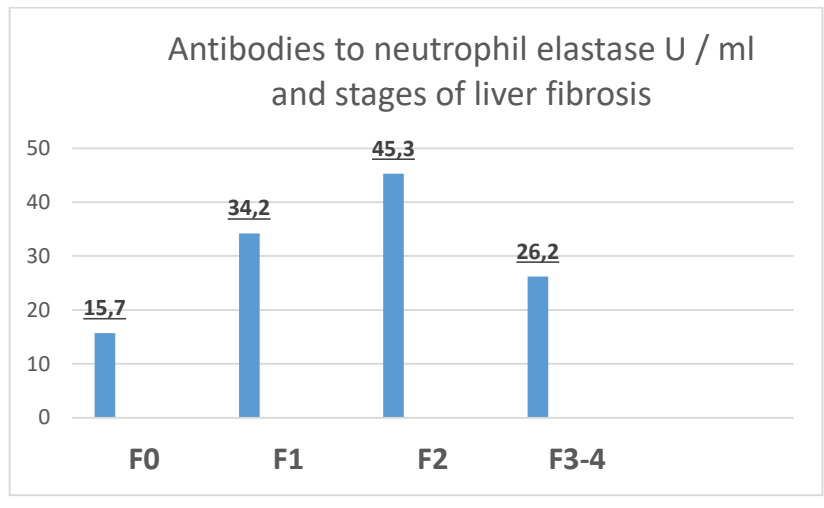

Fig. 1. Concentrations of antibodies to elastase depending on the stage of fibrosis

TIMP-1 levels also increase with the stage of fibrosis to F2 and are directly proportional to the concentration of antibodies to elastase $(r=0.46 ; \mathrm{p}<0.05)$ and do not depend on the frequency of COPD exacerbations.

The concentration of TGF- $\beta$ was significantly higher in patients with NAFLD + COPD with a predominance in group $1 \mathrm{~b}$, namely 3.4 times $(\mathrm{p}<0.05)$ more than in the control group and 2.2 times $(p<0,05)$ more than in patients of group 2. TGF- $\beta$ levels were significantly $(\mathrm{p}<0.05)$ increased in patients with F4 stage of liver fibrosis.

\section{Discussion}

Studies have shown that high levels of antibodies to neutrophil elastase reflect an increase in metabolic inflammation of the liver, thereby contributing to the progression of NAFLD. The concentration of antibodies to neutrophil elastase in the serum was associated with TNF-a, neopterin, C-reactive protein and some components of the metabolic syndrome. The results 
of this study showed that in patients with COPD comorbid with frequent exacerbations, the levels of CRP, IL-6, TNF- $\alpha$ and neopterin are significantly higher even in the remission phase of COPD, and than in patients who had one or did not tolerate any exacerbation for the last year. Stably high levels of markers of systemic inflammation in terms of TNF- $\alpha$, neopterin, CRP lead to the activation of TGF- $\beta$, which increases with the propgression of liver fibrosis. Activation of profibrotic cytokine -TGF- $\beta$ supports liver fibrosis, proving the role of COPD in the progression of NAFLD. The data obtained coincide with the results of other scientists who found a correlation between systemic inflammation and the progression of comorbidities in patients with COPD, which includes NAFLD (Barnes \& et al., 2009). The recorded consistently high concentration of $\mathrm{IgG}$ antibodies to neutrophil elastase may indirectly indicate a high level of activity of this enzyme in the serum in patients with NAFLD comorbid with COPD and confirms the participation of enzymatic activity of serum in liver fibrogenesis. Increased neutrophil elastase activity affects the activation of proteinase-activating receptors (PARs).

This leads to the start of the synthesis of transcriptional factors of connective tissue repair through reciprocal interaction between TGF- $\beta$ and EGFR-MEK-ERK signaling pathways, which is also an important factor in the development of tissue fibrosis (Hlapčić \& et al., 2020). In F3-4 fibrosis, the levels of IgG antibodies to neutrophil elastase are reduced, although they remain higher than the control values, which may indicate the attenuation of inflammatory processes in the liver and the predominance of fibrosis processes. Our data coincide with scientific reports that in patients with severe liver fibrosis (stage III, IV) elastase activity is significantly lower than in patients with initial stages, but higher than the level of control (Wu et al., 2020).

\section{Conclusion}

Levels of IgG antibodies to neutrophil elastase increase with increasing stage of liver fibrosis and are predictors of the development and progression of fibrotic changes in patients with NAFLD on the background of COPD. The concentration of antibodies to neutrophil elastase in the blood directly correlates with the levels of TNF-a, neopterin, CRP, ALT and with such components of the metabolic syndrome as body mass index, hypercholesterolemia and hypertriglyceridemia. Significant increase IgG antibody levels to neutrophil elastase depending on the stage of fibrosis and the activity of inflammatory changes indicates the role of serum enzymatic activity in the mechanisms of formation of more severe stages of NAFLD and can be considered as additional diagnostic markers.

\section{References}

Amulic, B., Cazalet, C., Hayes, G. L., Metzler, K. D., \& Zychlinsky, A. (2012). Neutrophil function: from mechanisms to disease. Annual review of immunology, 30, 459-489.

Barnes, P. J., \& Celli, B. R. (2009). Systemic manifestations and comorbidities of COPD. European respiratory journal, 33(5), 1165-1185.

Chan, S. M., Selemidis, S., Bozinovski, S., \& Vlahos, R. (2019). Pathobiological mechanisms underlying metabolic syndrome (MetS) in chronic obstructive pulmonary disease (COPD): clinical significance and therapeutic strategies. Pharmacology \& therapeutics, 198, 160-188. Hlapčić, I., Belamarić, D., Bosnar, M., Kifer, D., Vukić Dugac, A., \& Rumora, L. (2020). Combination of systemic inflammatory biomarkers in assessment of chronic obstructive pulmonary disease: Diagnostic performance and identification of networks and clusters. Diagnostics, 10(12), 1029. 
Huang, Y. L., Min, J., Li, G. H., Zheng, Y. Q., Wu, L. H., Wang, S. J., .. \& Mao, B. (2019). The clinical study of comorbidities and systemic inflammation in COPD. Sichuan da xue xue bao. Yi xue ban= Journal of Sichuan University. Medical science edition, 50(1), 88-108.

Lehman, H. K., \& Segal, B. H. (2020). The role of neutrophils in host defense and disease. Journal of Allergy and Clinical Immunology, 145(6), 1535-1544.

Liu, K., Wang, F. S., \& Xu, R. (2021). Neutrophils in liver diseases: pathogenesis and therapeutic targets. Cellular \& molecular immunology, 18(1), 38-44.

Wu, L., Gao, X., Guo, Q., Li, J., Yao, J., Yan, K., ...\& Guo, J. (2020). The role of neutrophils in innate immunity-driven nonalcoholic steatohepatitis: lessons learned and future promise. Hepatology International, 1-15.

Xu, Y., Zhang, Q., \& Zhao, Y. (2020). The functional diversity of neutrophils and clustered polarization of immunity. Cellular \& molecular immunology, 17(11), 1212-1214.

Yazici, O., Gulen, S. T., Yenisey, C., Eryilmaz, U., Abas, B. I., \& Polatli, M. (2020). Comparison of inflammation biomarkers among chronic obstructive pulmonary disease groups: a cross sectional study. Nigerian Journal of Clinical Practice, 23(6), 817. 